\title{
The composition of house dust mite is critical for mucosal barrier dysfunction and allergic sensitisation
}

\author{
S Post, ${ }^{1}$ M C Nawijn, ${ }^{1}$ T L Hackett, ${ }^{2}$ M Baranowska, ${ }^{1}$ R Gras, ${ }^{1}$ \\ A J M van Oosterhout, ${ }^{1}$ I H Heijink $^{1}$
}

- Additional materials are published online only. To view these files please visit the journal online (http://thorax.bmj. com/content/67/6.toc).

1 Laboratory of Allergology and Pulmonary Diseases, Department of Pathology and Medical Biology, University Medical Center Groningen, GRIAC Research Institute, University of Groningen, Groningen, The Netherlands ${ }^{2}$ James Hogg Research Centre, Department of Anaesthesiology, Pharmacology and Therapeutics, University of British Columbia, St Paul's Hospital, Vancouver, Canada

\section{Correspondence to} Professor A J M van Oosterhout, Laboratory of Allergology and Pulmonary Diseases, Department of Pathology and Medical Biology, University Medical Center Groningen (UMCG), Hanzeplein 1, NL-9713GZ Groningen, The Netherlands;

a.j.m.van.oosterhout@umcg.n

S Post and M C Nawijn contributed equally to the manuscript.

A J M van Oosterhout and I $\mathrm{H}$ Heijink share senior authorship.

Received 11 June 2011

Accepted 21 November 2011

Published Online First

13 December 2011

\begin{abstract}
Background House dust mite (HDM) allergens have been reported to increase airway epithelial permeability, thereby facilitating access of allergens and allergic sensitisation.

Objectives The authors aimed to understand which biochemical properties of HDM are critical for epithelial immune and barrier responses as well as T helper 2-driven experimental asthma in vivo.

Methods Three commercially available HDM extracts were analysed for endotoxin levels, protease and chitinase activities and effects on transepithelial resistance, junctional proteins and pro-inflammatory cytokine release in the bronchial epithelial cell line 16HBE and normal human bronchial cells. Furthermore, the effects on epithelial remodelling and airway inflammation were investigated in a mouse model.
\end{abstract}

Results The different HDM extracts varied extensively in their biochemical properties and induced divergent responses in vitro and in vivo. Importantly, the Greer extract, with the lowest serine protease activity, induced the most pronounced effects on epithelial barrier function and CCL20 release in vitro. In vivo, this extract induced the most profound epithelial E-cadherin delocalisation and increase in CCL20, CCL17 and interleukin 5 levels, accompanied by the most pronounced induction of HDMspecific lgE, goblet cell hyperplasia, eosinophilic inflammation and airway hyper-reactivity.

Conclusions This study shows the ability of HDM extracts to alter epithelial immune and barrier responses is related to allergic sensitisation but independent of serine/cysteine protease activity.

\section{INTRODUCTION}

Allergic asthma is characterised by allergen-specific IgE, T helper 2 (Th2)-mediated airway inflammation, airway remodelling and airway hyper-reactivity (AHR). The airway epithelium forms the first structural barrier against inhaled allergens. This epithelial barrier function is maintained by the formation of tight junctions (TJs), composed of zonula occludens (ZO) $1-3$, occludin and claudins $1-5$, as well as adherens junctions (AJs), consisting of E-cadherin, $\beta$-catenin and $\alpha$-catenin. Whereas TJs largely contribute to epithelial impermeability, E-cadherin is thought to provide the architecture required to form TJs. ${ }^{1}$

Many aeroallergens, including house dust mite (HDM), fungi and cockroach contain proteolytic activities. $^{2}{ }^{3}$ The HDM allergens Dermatophagoides

\section{Key messages}

What is the key question?

- The proteolytic activity of house dust mite allergens was hypothesised to be crucial for epithelial barrier dysfunction and subsequent activation of the innate immune response in asthma.

\section{What is the bottom line?}

- This study determined that the divergent abilities of these extracts to alter epithelial barrier and immune function in vitro are uniquely associated with the capacity to induce allergic sensitisation and asthma phenotypes in vivo.

\section{Why read on?}

- This makes the epithelial barrier an important target for future therapeutic strategies in asthma.

pteronyssinus (Der p) 1, 3, 6 and 9 are cysteine and/ or serine proteases, of which the serine peptidase activity has previously been reported to cleave ZO1 , occludin and to a lesser extent also E-cadherin. ${ }^{4}$ In addition to direct cleavage of junctional proteins, serine proteases can activate protease-activated receptor (PAR)-2, which can induce disruption of E-cadherin mediated cell-cell contacts. ${ }^{5}$ This may facilitate access of allergens to submucosal cells and promote allergic inflammation. ${ }^{67}$ In support of this notion, we have previously demonstrated that downregulation of E-cadherin in bronchial epithelium increases expression of the pro-allergic factors CCL17 and thymic stromal lymphopoietin (TSLP), which attract Th2 cells and promote Th2 cell differentiation respectively. ${ }^{8} 9$ Furthermore, PAR-2 activation by serine proteases induces activity of intracellular signalling pathways, including nuclear factor- $\mathrm{KB}$ (NF-KB), and subsequent release of the proinflammatory cytokines interleukin (IL)-6, IL-8, granulocyte macrophage colony-stimulating factor (GM-CSF) and TSLP in airway epithelium in vitro and in vivo. ${ }^{10} 11$ Based on studies in mouse models of asthma, the presence of proteases in HDM and subsequent PAR-2 activation are thought to play an important role in allergic sensitisation. ${ }^{12-14}$ In addition, a number of other biochemical activities and components of HDM, including chitin/chitinases, B-glucan and lipopolysaccharide (LPS), may contribute to allergic sensitisation. ${ }^{15-17}$ 
We hypothesised that proteolytic activity of HDM allergens is crucial for epithelial barrier dysfunction and subsequent activation of the innate immune response in asthma. We investigated different HDM extracts that vary extensively in biochemical properties and proteolytic activities, and assessed their effects on epithelial barrier function, release of pro-inflammatory cytokines and induction of Th2 responses, in vitro and in vivo. We demonstrate that the divergent abilities of these extracts to alter epithelial barrier and immune function in vitro are uniquely associated with the capacity to induce allergic sensitisation and asthma phenotypes in vivo. Of interest, this appeared to be independent of serine protease activity.

\section{METHODS}

See the online supplement for additional details.

\section{HDM extracts}

Three whole crushed body mite extracts were used. The first was kindly provided by Citeq Biologics (Groningen, The Netherlands), the other two were purchased from ALK-Abello (Abello, Spain) and from Greer Laboratories (Lenoir, North Carolina, USA). When indicated, HDM extracts were pretreated with the serine inhibitor 4-(2-aminoethyl) benzenesulfonyl fluoride hydrochloride (AEBSF) (Sigma, St Louis, Missouri, USA) or the cysteine inhibitor E-64 (Sigma) at concentrations of $0.1 \mathrm{mM}$ and $0.01 \mathrm{mM}$, respectively, for $30 \mathrm{~min}$ at $37^{\circ} \mathrm{C}$, or heat inactivated for $1 \mathrm{~h}$ at $95^{\circ} \mathrm{C}$.

\section{Cells}

The human bronchial epithelial cell line 16HBE14o- was kindly provided by Dr D C Gruenert (University of California, San Francisco, California, USA). Normal human bronchial epithelial (NHBE) cells were derived from Lonza (Walkersville, Maryland, USA). The cells were cultured as previously described ${ }^{8} 18$ and used as indicated for electric cell-substrate impedance sensing (ECIS), ELISA, immunodetection and immunofluorescent staining (see online data supplement).

\section{Electric cell-substrate impedance sensing}

Electrical resistance was measured using ECIS in a confluent monolayer of $16 \mathrm{HBE}$ cells, as previously described. ${ }^{19} 20$ The ECIS is a technique that allows for real-time quantitative monitoring of changes in resistance as measurement of cell-cell contacts and changes in capacitance as measurement of cell-matrix contacts. ${ }^{19}$ Resistance and capacitance to current flow were measured at frequencies of $400 \mathrm{~Hz}$ and $40 \mathrm{kHz}$ respectively (Applied Biophysics, Troy, New York, USA).

\section{Animals}

Male Balb/c mice (6-8 weeks) were purchased from Charles River Laboratories (L'Arbresle Cedex, France), kept under specific pathogen-free conditions and maintained on a $12 \mathrm{~h}$ light-dark cycle, with food and water ad libitum. Experiments were approved by the Institutional Animal Care and Use Committee of the University of Groningen (The Netherlands).

\section{HDM sensitisation protocol}

HDM extracts and LPS (Sigma) were dissolved in sterile phosphate-buffered saline (PBS; $2.5 \mathrm{mg}$ total weight/ml) and administered intranasally in $10 \mu \mathrm{l}$, twice weekly for 5 weeks. Mice were anaesthetised with isoflurane/oxygen (Nicholas Piramal India Ltd, London, UK). Twenty-four hours after the last sensitisation airway responsiveness was measured by Flexivent (SCIREQ, Montreal, Canada), lungs were lavaged and blood and lung tissues were collected.
Cytokine assay in cell supernatants and mouse lung tissue

Human CCL20 and GM-CSF protein was measured in cell-free supernatants from 16HBE cells and murine IL-5, IL-13, CCL20, thymus and activation-related chemokine (TARC), TSLP and GM-CSF was determined in cell-free supernatant from homogenised lung using Duoset ELISA Development Kit (R\&D Systems, Minneapolis, Minnesota, USA). The ELISAs were used according to the manufacturer's guidelines.

\section{Statistical analysis}

We assumed normal distribution and used the Student $t$ test for paired observations in the experiments with 16HBE. In animal experiments, the Mann-Whitney $U$ test was used.

\section{RESULTS \\ Disruption of bronchial epithelial cell-cell contacts upon exposure to the HDM extracts}

First, we tested the HDM extracts for proteolytic activity, chitinase levels and endotoxin content. Although we stratified the extracts on the basis of total proteolytic activity, analyses revealed that the extracts varied extensively in their other biochemical properties, including protein content (table 1). The Citeq and ALK extract contained the highest serine/cysteine protease activities, while levels of these proteases were relatively low in the Greer extract. Instead, this extract contained other, partially heat-insensitive proteases and the highest Der p2 content. ALK and Greer comprised substantial heat-sensitive exochitinase and endochitinase activity (table 1).

To test our hypothesis that the proteolytic activity of the HDM extracts is critical for airway epithelial barrier dysfunction, we exposed $16 \mathrm{HBE}$ cells to the different extracts in concentrations rendering equal levels of total proteolytic activity (see table 1). First, we evaluated the effect on occludin protein stability, since Der p1 has been shown to induce cleavage of occludin, leading to increased permeability of the epithelial layer. ${ }^{7}$ Exposure to all HDM extracts induced smaller molecular weight cleavage products of $\sim 37 \mathrm{kD}$ and $\sim 25 \mathrm{kD}$, as shown by immunodetection (figure 1A), although the latter fragment was not observed with the Citeq extract. A similar degradation pattern was previously described by Wan et al, when epithelial cells were exposed to Der p1. ${ }^{7}$ Furthermore, the Greer extract yielded an additional fragment of $\sim 45 \mathrm{kD}$, which was also observed upon exposure to a protease cocktail used as positive control. Importantly, the appearance of degradation fragments could not be blocked by treatment of the extracts with serine protease inhibitor AEBSF, cysteine protease inhibitor E64 or heat inactivation (figure $1 \mathrm{~A}$ ), suggesting that these effects of HDM can occur independently of serine/cysteine protease or chitinase activity.

To directly determine the effect of HDM on epithelial barrier function, we measured electrical resistance of $16 \mathrm{HBE}$ cell monolayers using ECIS. Exposure to the Greer, but not Citeq or ALK, extract induced a transient fall in epithelial resistance, with a maximum effect at $\sim 10-20 \mathrm{~min}$ and recovery to baseline values within $60 \mathrm{~min}$ (figure $1 \mathrm{~B}$ ). This effect could not be inhibited by heat inactivation or pretreatment of the extract with the serine/cysteine protease inhibitors (figure 1C).

Prolonged exposure $(24 \mathrm{~h})$ to the Citeq extract, but not the other HDM extracts, dramatically decreased epithelial resistance (online supplementary figure $2 \mathrm{~A}$ ), which was parallelled by detachment of the cells (online supplementary figure $2 \mathrm{~B}$ ) and attenuated by treatment of the extract with the serine protease inhibitor (data not shown). LPS, which was present in our 
Table 1 Biochemical properties of the house dust mite (HDM) extracts in the concentration applied in vitro

\begin{tabular}{|c|c|c|c|}
\hline & Citeq & ALK & Greer \\
\hline Der p1 content (ng) & 6.25 & 50 & 81 \\
\hline Der p2 content (ng) & 1 & 22.2 & 428.9 \\
\hline Protein content $(\mu \mathrm{g} / \mathrm{ml})$ & 2.64 & 12 & 50 \\
\hline Total protease activity (U/ml) & $5 \pm 0.5$ & $5 \pm 1.5$ & $5 \pm 0.7$ \\
\hline Protease activity after $\mathrm{HI}(\mathrm{U} / \mathrm{ml})$ (\% of total) & $0.9 \pm 0.2(18 \%)$ & $1.6 \pm 0.2(32 \%)$ & $1.4 \pm 0.3(28 \%)$ \\
\hline $\begin{array}{l}\text { Protease activity remaining after AEBSF } \\
\text { treatment }(\mathrm{U} / \mathrm{ml})(\% \text { of total })\end{array}$ & $2 \pm 0.3(40 \%)$ & $4.2 \pm 1.4(84 \%)$ & $5 \pm 0.6(99.9 \%)$ \\
\hline $\begin{array}{l}\text { Protease activity remaining after E64 } \\
\text { treatment }(\mathrm{U} / \mathrm{ml}) \text { (\% of total) }\end{array}$ & $4 \pm 0.9(80 \%)$ & $5 \pm 1.9(99.9 \%)$ & $4.8 \pm 0.8(96 \%)$ \\
\hline Serine protease activity $\left(10^{3}\right.$ mean $\left.\mathrm{V} / \mathrm{ml}^{*}\right)$ & $2222 \pm 45$ & $2087 \pm 5$ & $162.7 \pm 58$ \\
\hline $\begin{array}{l}\text { Serine protease activity remaining after AEBSF } \\
\text { treatment }\left(10^{3} \text { mean } \mathrm{V} / \mathrm{ml}^{*}\right)\end{array}$ & 0 & 0 & 0 \\
\hline Cysteine protease activity $\left(10^{3}\right.$ mean $\left.\mathrm{V} / \mathrm{ml}^{*}\right)$ & $892 \pm 1$ & $1191 \pm 14$ & $95 \pm 2$ \\
\hline $\begin{array}{l}\text { Cysteine protease activity remaining after E64 } \\
\text { treatment }\left(10^{3} \text { mean } \mathrm{V} / \mathrm{ml}^{*}\right) \text { ( } \% \text { of total) }\end{array}$ & $468 \pm 6(52.4 \%)$ & $747 \pm 3(62.7 \%)$ & $80 \pm 2(84 \%)$ \\
\hline $\begin{array}{l}\text { Exochitinase (ß-N-acetylglucosaminidase) } \\
\text { levels }\left(10^{-6} \mathrm{U} / \mathrm{ml}\right)\end{array}$ & $2 \pm 0.5$ & $2400 \pm 4$ & $1800 \pm 120$ \\
\hline $\begin{array}{l}\text { Exochitinase (ß-N-acetylglucosaminidase) } \\
\text { levels after } \mathrm{HI}\left(10^{-6} \mathrm{U} / \mathrm{ml}\right)\end{array}$ & $0.4 \pm 0.4$ & $18 \pm 0.6$ & $60 \pm 0.6$ \\
\hline Exochitinase (chitobiosidase) levels $\left(10^{-6} \mathrm{U} / \mathrm{ml}\right)$ & $1 \pm 0.03$ & $800 \pm 6$ & $300 \pm 18$ \\
\hline Exochitinase (chitobiosidase) levels after $\mathrm{HI}\left(10^{-6} \mathrm{U} / \mathrm{ml}\right)$ & $1 \pm 0.1$ & $18 \pm 0.6$ & $120 \pm 4.8$ \\
\hline Endochitinase levels $\left(10^{-6} \mathrm{U} / \mathrm{ml}\right)$ & $1 \pm 0.03$ & $400 \pm 4$ & $300 \pm 18$ \\
\hline Endochitinase levels after $\mathrm{HI}\left(10^{-6} \mathrm{U} / \mathrm{ml}\right)$ & $1 \pm 0.01$ & $20 \pm 2$ & $60 \pm 3$ \\
\hline Endotoxin (LPS) level (EU/ml) & 7.1 & 0.28 & 31.65 \\
\hline
\end{tabular}

extracts (see table 1), did not affect epithelial barrier function at the highest concentration found in the extracts $(50 \mathrm{U} / \mathrm{ml}$; data not shown).

Next, we visualised the effects of the HDM extracts on TJ and AJ integrity. Using immunofluorescent staining, we observed continuous circumferential localisation of E-cadherin, ZO-1 and occludin at the cell membrane of the $16 \mathrm{HBE}$ cells at baseline conditions (figure 1D). Exposure to all three HDM extracts (15 min) induced delocalisation of E-cadherin, ZO-1 and occludin from the membrane. In line with the Greer-induced epithelial barrier dysfunction, these effects were most pronounced upon exposure to the Greer extract (figure 1D). In accordance with the effects on occludin cleavage (figure 1A), heat inactivation of the HDM extracts did not abrogate the delocalisation of occludin, ZO-1 and E-cadherin (online supplementary figure 1). To verify our results in primary cells, we also studied the effects of the HDM extracts on NHBE cells. Here, all $\mathrm{HDM}$ extracts induced marked delocalisation of E-cadherin, ZO-1 and occludin (figure 1E). Again, the Greer extract induced the most pronounced effect on E-cadherin and occludin, although this was not clearly the case for ZO-1.

In summary, all extracts induced delocalisation of junctional proteins to some extent, with the most pronounced effect of the Greer extract, which also induced a transient decrease in epithelial resistance in $16 \mathrm{HBE}$ cells.

\section{Cytokine levels upon exposure of bronchial epithelial cells to the HDM extracts}

HDM can induce epithelial expression of pro-inflammatory cytokines and chemokines, including CCL20 and GM-CSF. ${ }^{10} 1721$ CCL20 is known to attract naïve dendritic cells towards the airway mucosa, ${ }^{22}$ while GM-CSF induces maturation and acti- vation of these cells. ${ }^{23}$ The Greer extract, but not the Citeq or ALK extracts, induced a strong and significant increase ( fourfold) in CCL20 levels in 16HBE cells, which was not significantly affected by heat treatment of the extract (figure $2 \mathrm{~A}$ ). The secretion of GM-CSF was slightly, but not significantly increased upon exposure to all extracts, and again not affected upon heat inactivation (figure $2 \mathrm{~B})$. Furthermore, LPS $(50 \mathrm{EU} / \mathrm{ml}$ ) did not affect CCL20 or GM-CSF secretion by $16 \mathrm{HBE}$ cells (data not shown). Additional experiments with NHBE cells showed that both the Citeq and Greer extract induced a significant increase in CCL20 levels, while GM-CSF levels were also significantly enhanced after exposure to the Greer extract (figure 2C,D).

\section{In vivo responses to the HDM mite extracts}

Next, we tested which of the HDM extracts was able to induce airway inflammation in vivo. Here, mice received $10 \mu \mathrm{l}$ of $2.5 \mathrm{mg} / \mathrm{ml} \mathrm{HDM}$ extract (see table 2) or $10 \mu \mathrm{l} \mathrm{PBS}$ at each administration. Of note, HDM extracts were administered based on total weight and not on protein content or on total protease content (as in the in vitro experiments). Administration of all HDM extracts induced delocalisation of E-cadherin in airway epithelium compared with the PBS-treated mice, with the most pronounced effect of the Greer extract (figure 3A,B). Interestingly, these effects could already be observed after a single administration of the HDM extracts to naïve mice (data not shown). Importantly, the Greer extract, but none of the other extracts, also induced substantial goblet cell hyperplasia (figure $3 \mathrm{~A}$ ).

Interestingly, Greer extract-treated mice, but not mice treated with the other extracts, displayed significantly increased levels of CCL20 and CCL17 in lung tissue compared with PBS-treated 
A

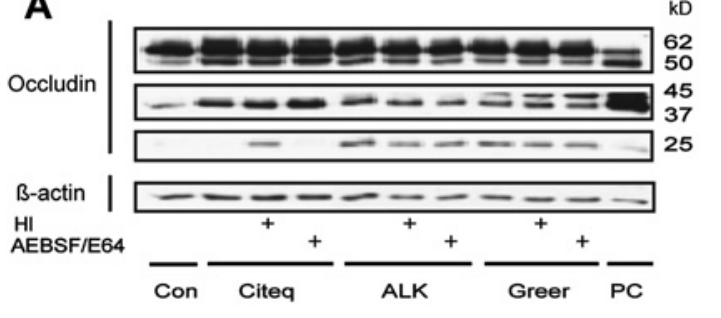

B

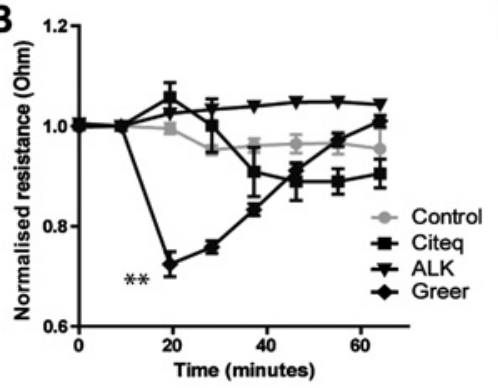

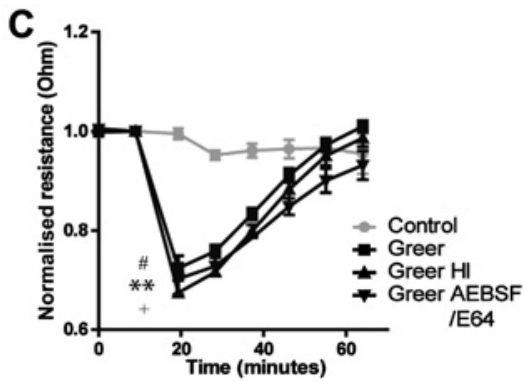

D

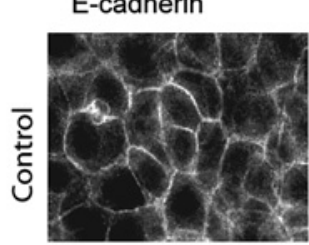

ZO-1
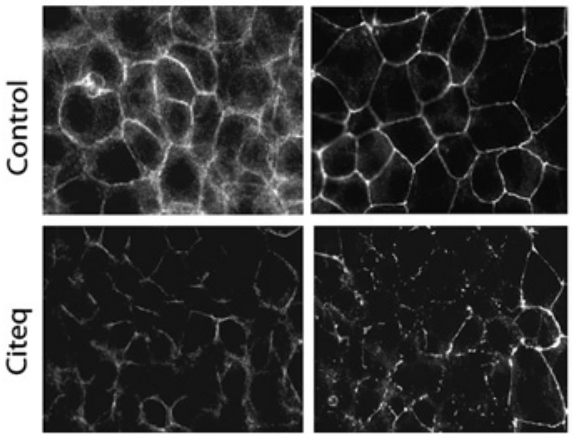

Occludin
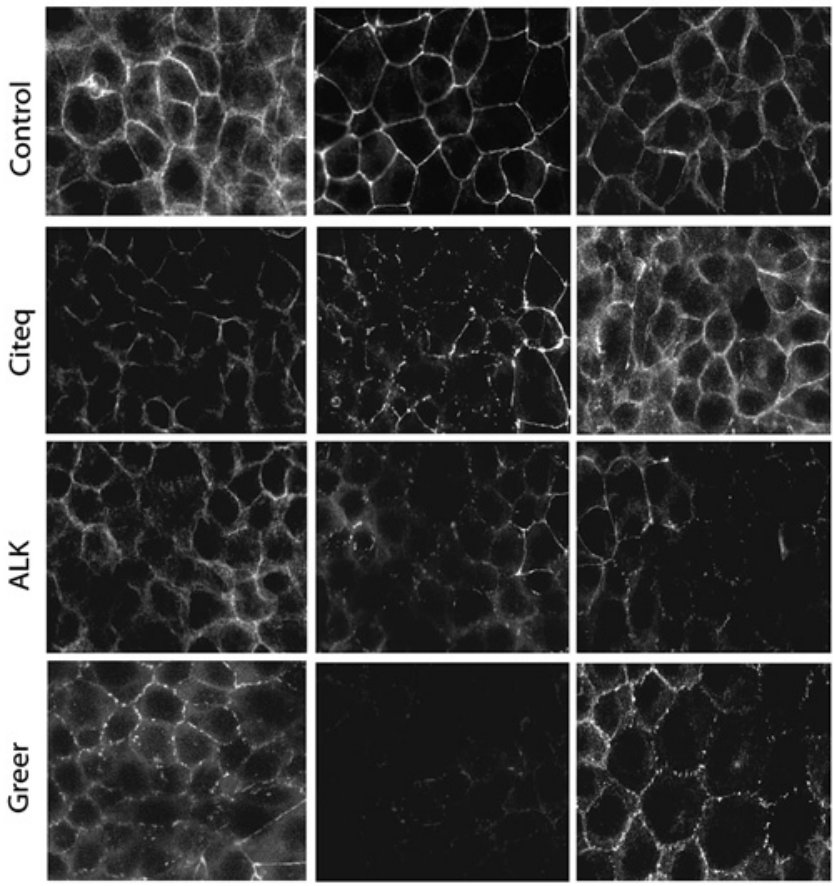

E

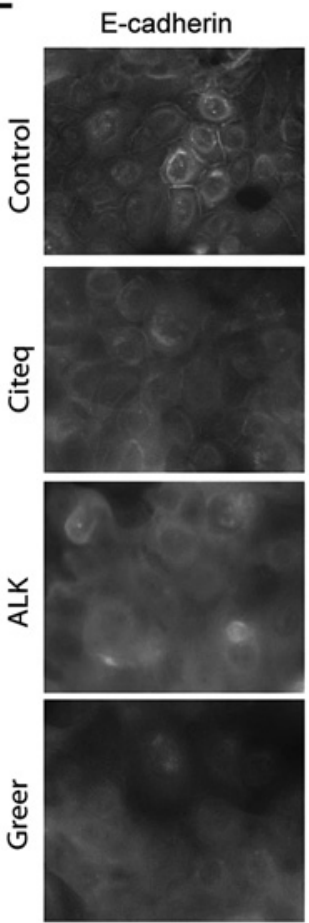

$\mathrm{ZO}-1$

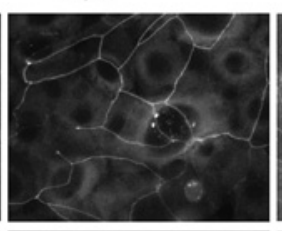

Occludin
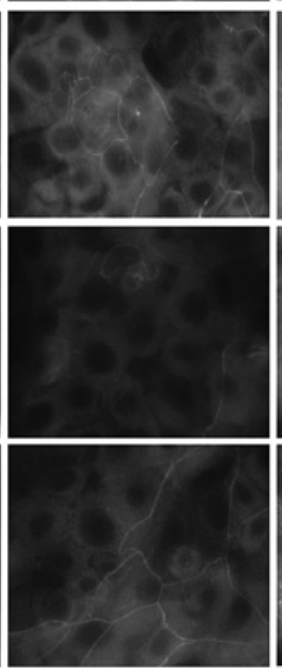

Figure 1 Effects of the house dust mite (HDM) extracts on tight and adherens junctions in human bronchial epithelium. 16HBE cells were seeded in duplicates, grown for 3-5 days in 24-well plates, LabTeks or electric cell-substrate impedance sensing arrays, serum deprived overnight and incubated with or without a protease cocktail (PC; $2.5 \mu \mathrm{g} / \mathrm{ml}$ ) or HDM extracts that were treated with or without 4-(2-aminoethyl) benzenesulfonyl fluoride hydrochloride (AEBSF) $(0.1 \mathrm{mM})$, E64 $(0.01 \mathrm{mM})$ or heat inactivated $(\mathrm{HI})\left(95^{\circ} \mathrm{C}, 1 \mathrm{~h}\right) .(\mathrm{A})$ Total cell lysates were prepared and occludin was detected by immunoblotting. B-actin was used as a control (con) for equal loading. A representative from three independent experiments is shown. (B) Resistance was measured immediately after $\mathrm{HDM}$ administration at $400 \mathrm{~Hz}$ and normalised to the values prior to HDM administration. Mean levels $( \pm$ SEM) are shown $(n=3)$. (C) Resistance was measured immediately after HDM administration at $400 \mathrm{~Hz}$ and normalised to the values prior to $\mathrm{HDM}$ administration, compared with AEBSF/E64-treated or heat-inactivated HDM. Mean levels ( \pm SEM) are shown $(n=3)$. ${ }^{* *} p<0.01$ between control and Greer-treated 16HBE cells, $\# p<0.001$ between control and heat-inactivated Greer-treated $16 \mathrm{HBE}$ cells, and $+p<0.05$ between control and AEBSF/ E64-treated Greer-treated 16HBE cells. (D) E-cadherin, zonula occludens (ZO)-1 and occludin were detected 15 min after stimulation with or without (control) HDM in 16HBE cells by immunofluorescent staining. Representatives from three independent experiments are shown. (E) E-cadherin, ZO-1 and occludin were detected 15 min after stimulation with or without (control) HDM in normal human bronchial epithelial cells by immunofluorescent staining. Representatives from three independent experiments are shown.

mice (figure 3C,D). None of the HDM extracts significantly altered TSLP or GM-CSF levels $24 \mathrm{~h}$ after the last application (figure $3 \mathrm{E}, \mathrm{F}$ ). Furthermore, lung cytokine/chemokine levels were not significantly altered upon administration of an equivalent amount of LPS (figure 3C-F; see also table 3 in the online supplement for absolute values). In addition, Eotaxin- 1 and $\mathrm{KC}$ levels were increased after exposure to Greer and to a lesser extent also to the Citeq extract, while exposure to the Citeq extract also increased the levels of IL-17 (online supplementary figure 3).

To assess whether mucosal application of the different HDM extracts induced allergic sensitisation in vivo, we analysed the HDM-specific IgE responses. Both the Citeq and Greer, but not
ALK, extract, induced a significant increase in HDM-specific IgE levels (figure 4A), while only the Citeq extract significantly increased total IgE levels (figure $4 \mathrm{~B}$, see also table 3 in the online supplement for absolute values). To confirm that HDM-specific IgE levels were sufficient to induce an immediate allergic response, we measured the ear swelling response $2 \mathrm{~h}$ after local HDM injection in the HDM-treated mice. We observed a significant increase in ear thickness in Greer extract-treated mice (figure $4 \mathrm{C}$ ) and a trend in Citeq extract-treated mice $(p=0.055)$, indicating that both HDM extracts were able to induce allergic sensitisation via the airways.

Additionally, treatment with the Greer extract induced a significant increase in lung IL-5 (figure 4D), while the Citeq 
Figure 2 Pro-inflammatory cytokine production upon exposure of $16 \mathrm{HBE}$ cells to the house dust mite (HDM) extracts. Cells were seeded in duplicates, grown to confluence, serum deprived overnight and left unstimulated (control, con) or stimulated with heatinactivated (HI) or untreated HDM extracts. Cytokines were measured in cell-free supernatants. (A) Levels of CCL20 after $24 \mathrm{~h}$ of stimulation with or without HDM extract in 16HBE cells.

(B) Levels of granulocyte macrophage colony-stimulating factor (GM-CSF) after $48 \mathrm{~h}$ of stimulation with or without HDM extract in 16HBE cells. (C) Levels of CCL20 $24 \mathrm{~h}$ with and without HDM extract in normal human bronchial epithelial (NHBE) cells. (D) Levels of GM-CSF $24 \mathrm{~h}$ after stimulation with and without HDM extract in NHBE cells. Mean absolute levels $( \pm S E M)$ are shown $(n=3) .{ }^{*} p<0.05$ between control and HDM-treated 16HBE cells.
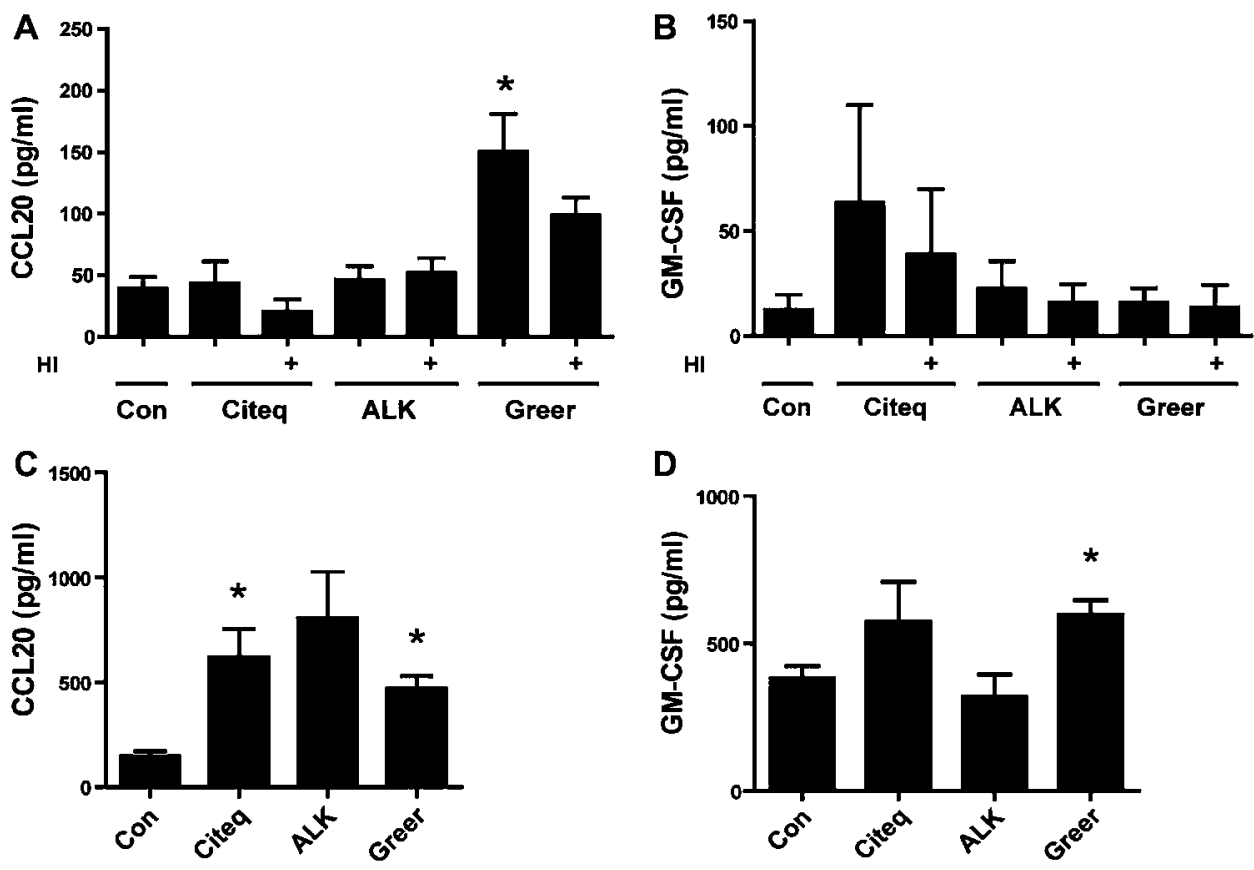

extract induced increased levels of IL-13 (figure 4E; see also table 3 in the online supplement for absolute values). Treatment with the ALK extract or LPS did not induce a significant increase in these Th2 cytokines (figure 4D,E). In accordance with the increase in IL-5, exposure to the Greer extract, but none of the other extracts, induced lung inflammatory cell recruitment as evaluated by haematoxylin eosin staining (figure 5A). Quantification of the profile of the inflammatory cells in bronchoalveolar lavage (BAL) of Greer-extract treated mice revealed that the increased eotaxin and KC levels were indeed accompanied by increased numbers of eosinophils and neutrophils (figure $5 \mathrm{~B}$ ). Since the Greer extract was the only extract to induce airway inflammation, we aimed to confirm that this HDM extract was also able to induce AHR. As shown in figure 5C, the Greer extract induced an increase in AHR to metacholine compared with PBS-treated control mice, which only reached significance at the highest dose of metacholine.

Table 2 Biochemical properties of the house dust mite (HDM) extracts in the concentration applied in vivo

\begin{tabular}{llll}
\hline & Citeq & ALK & Greer \\
\hline Der p1 (ng) & 100 & 97 & 16.70 \\
Der p2 (ng) & 16 & 43 & 88.36 \\
Total protease activity (U/ml) & $5020.17 \pm 30$ & $253.8 \pm 74$ & $75.8 \pm 11$ \\
$\begin{array}{l}\text { Serine protease activity } \\
\left(10^{6} \text { mean } \mathrm{V} / \mathrm{ml}^{*}\right)\end{array}$ & $2221.9 \pm 44.8$ & $104.4 \pm 0.3$ & $2.7 \pm 1$ \\
$\begin{array}{l}\text { Cysteine protease activity } \\
\left(10^{6} \mathrm{mean} \mathrm{V} / \mathrm{ml}^{*}\right)\end{array}$ & $891.9 \pm 1.3$ & $59.6 \pm 0.7$ & $31.5 \pm 2.5$ \\
$\begin{array}{l}\text { Exochitinase (B-N-acetylglucosaminidase) } \\
\text { levels }\left(10^{-3} \mathrm{U} / \mathrm{ml}\right)\end{array}$ & $2.3 \pm 0.3$ & $124.9 \pm 2.2$ & $27.2 \pm 1.4$ \\
$\begin{array}{l}\text { Exochitinase (chitobiosidase) levels } \\
\left(10^{-3} \mathrm{U} / \mathrm{ml}\right)\end{array}$ & $1 \pm 0.03$ & $39.7 \pm 0.4$ & $5.2 \pm 0.03$ \\
Endochitinase levels $\left(10^{-3} \mathrm{U} / \mathrm{ml}\right)$ & $1.3 \pm 0.03$ & $20 \pm 0.2$ & $5 \pm 0.3$ \\
Endotoxin (LPS) level (EU/ml) & 7100 & 14 & 527.5
\end{tabular}

The table shows several aspects of the biochemical properties found in the HDM extracts $(2.5 \mathrm{mg} / \mathrm{ml})$ when tested on BALB/c mice.

*Arbitrary unit.

Der $\mathrm{p}$, Dermatophagoides pteronyssinus; EU, endotoxin unit; HDM, house dust mite; LPS, lipopolysaccharide; U, unit.
In summary, the extract that exerted the most pronounced effects on epithelial immune and barrier function in vitro and on epithelial remodelling in vivo also induced the most profound allergic responses in our mouse model.

\section{DISCUSSION}

The airway epithelial barrier is an important target for the proteolytic activities of allergens and may play a crucial role in allergic sensitisation. ${ }^{24}$ Disruption of the epithelial barrier may facilitate transport of allergens to allergen-presenting cells and promote pro-inflammatory activities of the epithelium. ${ }^{142125}$ In this study we investigated the effects of three different HDM extracts, varying extensively in composition and proteolytic activities. Interestingly, the Greer extract, which exerted the most pronounced effects on epithelial immune and barrier function in vitro, also induced allergic sensitisation and manifestations of asthma, including goblet cell hyperplasia, inflammatory cell infiltrates and increased Th2 cytokine levels in vivo. Importantly, this extract displayed the lowest serine and cysteine protease activity. Furthermore, the in vitro effects could not be prevented by heat inactivation and could not be mimicked by LPS administration. In line with our observations, De Alba et al have shown that the Greer extract was still able to induce manifestations of asthma upon heat inactivation in a rat model. ${ }^{26}$ Our data show for the first time that serine/cysteine proteases and chitinases in HDM extracts are not critically required for disruption of epithelial barrier function in vitro and innate immune responses in vivo, and hence subsequent allergic sensitisation and eosinophilic airway inflammation.

The Greer and Citeq extracts, which displayed the lowest and the highest serine protease activity respectively, induced HDMspecific IgE levels and an immediate allergic response in vivo, as measured by ear swelling upon topical application of the allergen. These data indicate that an IgE response can be induced independently of serine protease activity, in line with a previous report in which mice were sensitised by intratracheal aspiration with either protease-active or protease-depleted German cockroach faeces extract. ${ }^{27}$ Remarkably, the same group reported that protease activity did have an effect on serum IgE when the 
A Control

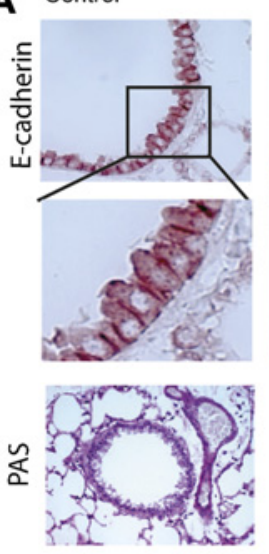

Citeq
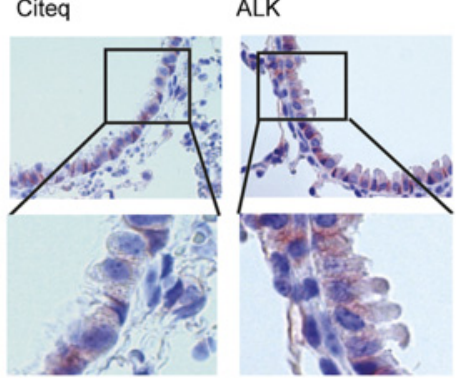

Greer

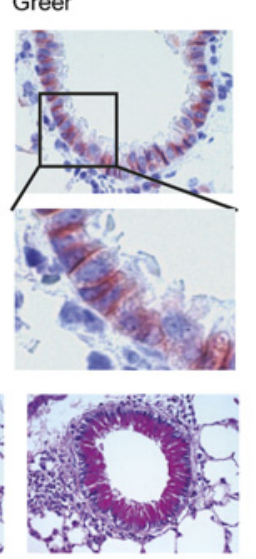

B

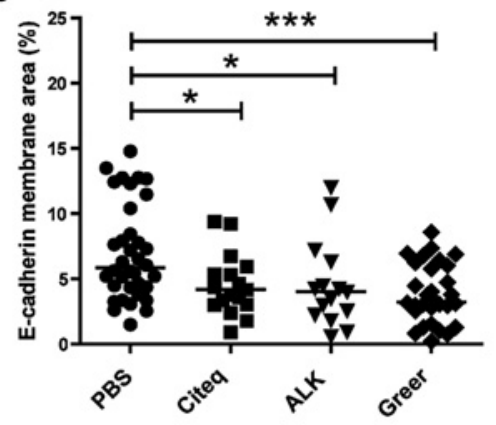

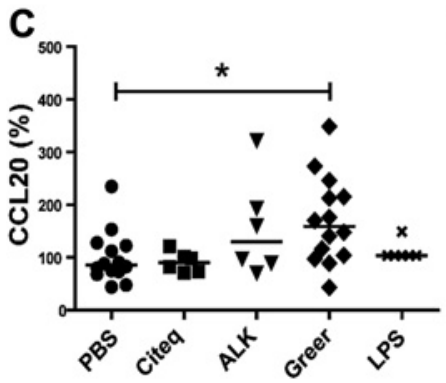
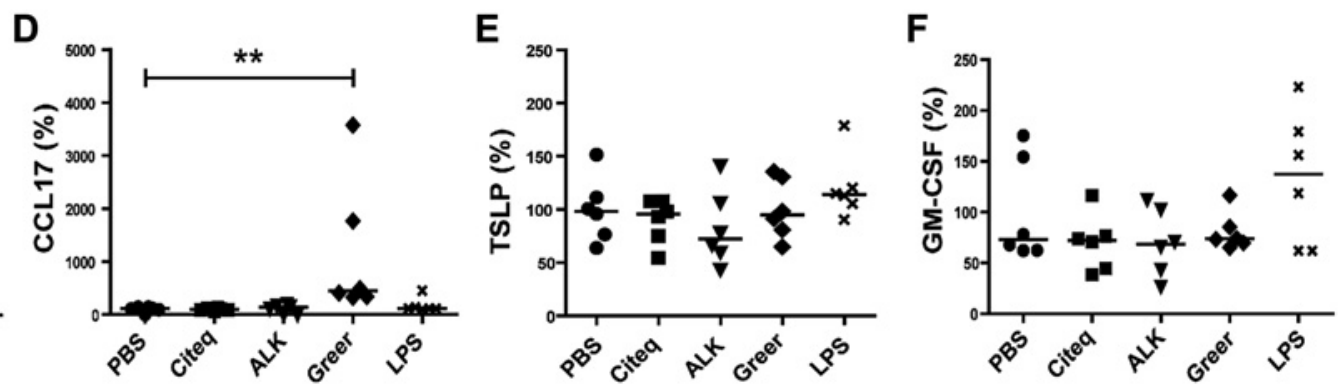

Figure 3 Effect of the house dust mite (HDM) extracts in a mouse model of asthma. Balb/c mice ( $n=6-14$ per group) were exposed to $10 \mu l$ of different HDM extracts $(2.5 \mathrm{mg} / \mathrm{ml})$, lipopolysaccharide (LPS) $(2.5 \mathrm{mg} / \mathrm{ml})$ or phosphate-buffered saline (PBS) twice a week for 5 weeks. Mice were sacrificed $24 \mathrm{~h}$ after the final intranasal challenge. Lung sections were stained for (A) E-cadherin and peroxidase-acid Schiff (PAS). Representative pictures are shown (original magnification $\times 40$ ). (B) Measurement of E-cadherin positive membrane staining (\%) analysed by Image-Pro Plus. Levels were expressed as percentage of E-cadherin staining on the membrane of the airway epithelium, medians are shown. ELISA measurements of (C) CCL20, (D) CCL17, (E) thymic stromal lymphopoietin and (F) granulocyte macrophage colony-stimulating factor (GM-CSF) in homogenised lung tissue, $24 \mathrm{~h}$ after the final intranasal challenge. Values were normalised to total protein content and expressed as percentages of control values. Relative levels and medians are shown. ${ }^{*} \mathrm{p}<0.05,{ }^{* *} \mathrm{p}<0.01$ and ${ }^{* * *} \mathrm{p}<0.001$ between HDM-treated and PBS-treated mice.

same allergen extracts were precipitated on alum and applied intraperitoneally, ${ }^{3}$ indicating that the relevance of the protease activity for the induction of an IgE response might depend on the context in which the allergen is presented. The Citeq extract also induced an increase in IL-13 and IL-17 in the lungs, which might contribute to the $\operatorname{IgE}$ response induced by Citeq. ${ }^{28}$

Figure 4 The allergic sensitisation response after house dust mite (HDM) exposure. Balb/c mice $(n=6-8$ per group) were exposed to $10 \mu \mathrm{HDM}$ extracts $(2.5 \mathrm{mg} / \mathrm{ml})$,

lipopolysaccharide (LPS) $(2.5 \mathrm{mg} / \mathrm{ml})$ or phosphate-buffered saline (PBS) twice a week for 5 weeks. (A) ELISA measurements of HDM-specific $\lg \mathrm{E}$, shown as absolute values. (B) Total IgE in mouse serum. Levels are expressed as percentages of control values and medians are shown. (C) lgE-dependent immediate allergic response measured by ear thickness $(\mathrm{mm})$ after intracutaneous injection of $25 \mu \mathrm{g} / \mathrm{ml}$ HDM in the right ear and PBS as control in the left ear. Absolute values and medians are shown. (D) Interleukin (IL)5 levels in homogenised lung tissue, $24 \mathrm{~h}$ after the final intranasal challenge. Values are normalised to total protein content and expressed as percentages of control values. Relative levels and medians are shown. (E) IL-13 levels in ho
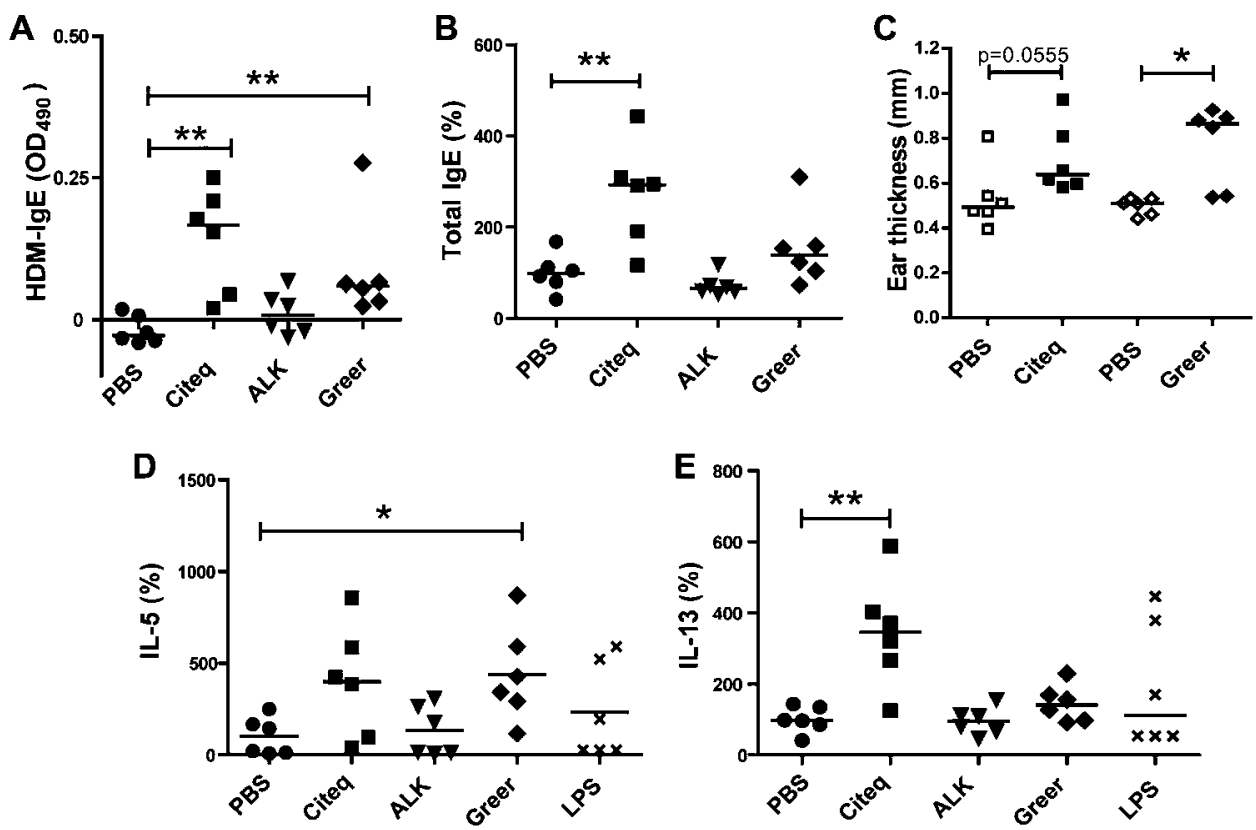
and expressed as percentages of control values. Relative levels and medians are shown. ${ }^{*} p<0.05$ and ${ }^{* *} p<0.01$ on comparison between HDMtreated and PBS-treated mice. 
Figure 5 The inflammatory response after allergen exposure. Balb/c mice ( $n=6-8$ per group) were exposed to $10 \mu$ phosphate-buffered saline (PBS), Citeq, ALK and/or Greer extract as indicated $(2.5 \mathrm{mg} / \mathrm{ml})$ twice a week for 5 weeks. Mice were sacrificed $24 \mathrm{~h}$ after the final intranasal challenge. (A) Lung sections were stained for haematoxylin eosin. Representative pictures are shown (magnification $\times 40$ ). (B) Total, mononuclear, eosinophiland neutrophil numbers were determined in bronchial airway lavage fluid (BALF). Absolute numbers and medians are shown. (C) Airway hyperreactivity was measured by Flexivent. Absolute mean values ( \pm SEM) are shown. ${ }^{*} p<0.05$ and ${ }^{* * *} \mathrm{p}<0.001$ on comparison between HDM-treated and PBS-treated mice.
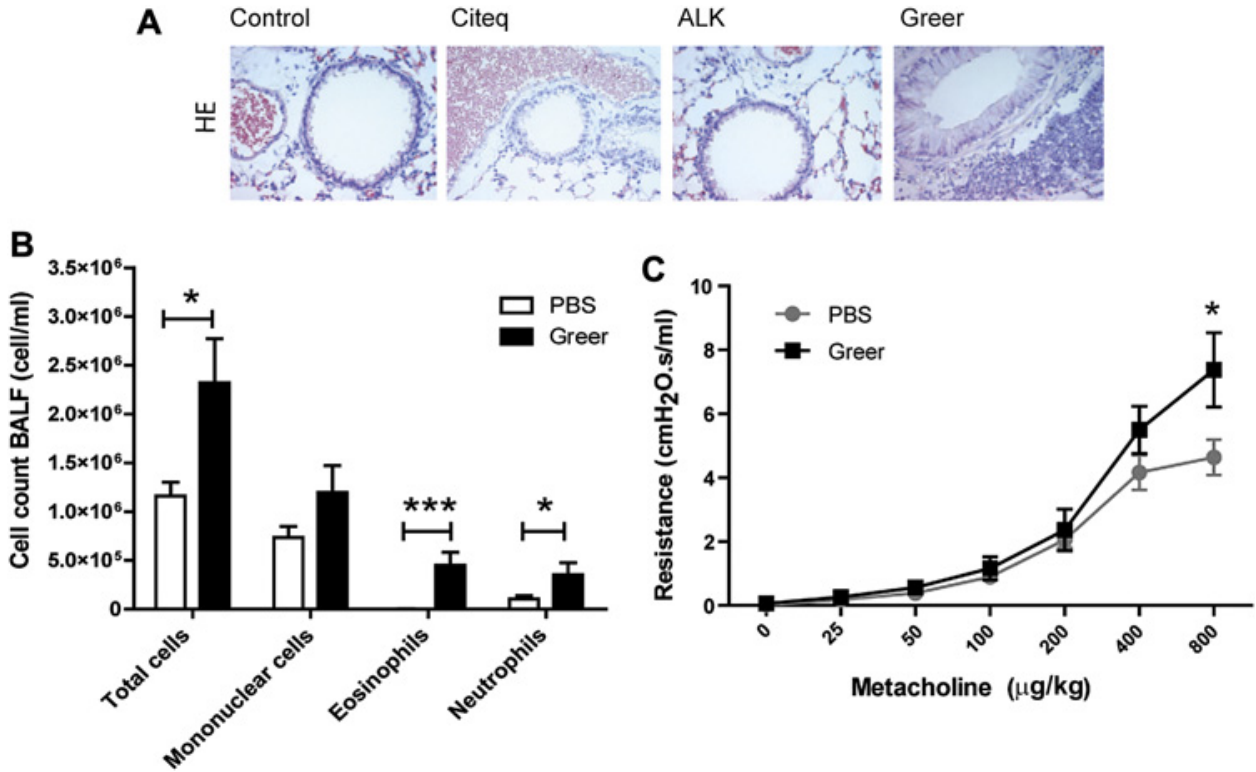

Importantly, we observed that treatment with the Greer extract, containing the lowest serine protease activity, increased HDM-specific IgE levels and induced goblet cell hyperplasia, delocalisation of E-cadherin, profound (eosinophilic) airway inflammation, AHR and increased KC, CCL17, Eotaxin-1 and IL-5 levels in the lungs. In contrast, the Greer extract did not induce substantial secretion of IL-17, indicating that the $\mathrm{KC}$ production induced by this extract is likely responsible for recruitment of the neutrophils into the BAL. The widely divergent responses induced by the three different HDM extracts reflect the remarkable differences in biochemical composition between the extracts (see table 1), precluding a straightforward association of their individual properties to the induction of a defined biological response in vitro or in vivo. However, we observed a very interesting and highly relevant positive association between several biological responses induced by a single HDM extract. The Greer extract induced loss of barrier function and pro-inflammatory responses in vitro, and allergic sensitisation, airway remodelling, AHR and eosinophilic inflammation in vivo, suggesting a putative causal relationship between airway epithelial responses and the induction of a Th2-polarised immune response.

The induction of the above-mentioned asthma manifestations appears to be independent of serine/cysteine proteases, chitinase activities and LPS levels. Our data show that these manifestations are induced by the Greer extract that uniquely decreased epithelial barrier function and induced the most profound delocalisation of occludin, ZO-1 and E-cadherin, an effect that is independent of heat inactivation of the extract. Furthermore, the Greer extract was still proteolytically active and able to cleave occludin upon heat inactivation. Thus, an unidentified heat-insensitive protease might contribute to the disruption of epithelial TJ proteins. The intracellular protein ZO-1 and the more basolaterally positioned E-cadherin were also delocalised upon exposure to all HDM extracts, indicating involvement of intracellular processes, for instance activation of PAR-2 receptors, ${ }^{5}$ known to be induced by serine proteases. Alternatively, activation of pattern recognition receptors may indirectly disrupt epithelial junctions. HDM extracts contain microbiological glucose structures, for example $\beta$-glucan, which can activate epithelial C-type lectin receptors, including dectin-1. ${ }^{17}$
Activation of these receptors induces $\mathrm{Ca}^{2+}$ fluxes ${ }^{29}$ and we speculate that this may lead to cleavage of cell-cell contact proteins by activation of the endogenous protease calpain. ${ }^{30}$ It has also been described that activation of the dectin- 1 receptor induces rapid secretion of CCL20 by $16 \mathrm{HBE}$ cells. ${ }^{17}$ We only observed a substantial increase in CCL20 levels in vitro and in vivo upon exposure to the Greer extract. Thus, it will be of interest to further study the role of $\beta$-glucan in the effects of HDM extracts on epithelial immune barrier function.

Next to proteolytic allergens, HDM contains Der p2, a nonproteolytic allergen, which is structurally homologue to protein associated with the TLR4ecto-domain (MD-2). This enables its interaction with toll-like receptor-4 (TLR4), which may facilitate airway inflammation. ${ }^{31}$ Interestingly, the Greer extract with the most pronounced effects on epithelial barrier function contained the highest Der p2 levels. However, previously the effects of Der p2 on bronchial epithelial cells in vitro have been shown to disappear upon heat inactivation, ${ }^{32}$ whereas our observed effects remained upon heat treatment. Thus a role for Der $\mathrm{p} 2$ in the observed effects seems unlikely. In addition to the potential activation of TLR4 by Der p2, biochemical analysis revealed that all three HDM extracts contained substantial levels of LPS, which can also activate TLR4 (see table 1). However, LPS exposure did not mimic the effects on barrier function in vitro or inflammatory responses in vivo, although we cannot exclude a role for a synergistic interaction between TLR4 and PAR-2. In addition, the LPS content in the HDM extracts did not correlate with neutrophil recruitment, although this might have been expected. $^{33}$ Finally, chitins as well as the activity of chitinases present in allergen extracts have been suggested to play a role in asthma. ${ }^{15}$ The effects observed in our study are not likely due to chitinases, since chitinase activity could be blocked by heat inactivation (see table 1). Future studies will have to identify which specific HDM components are responsible for the effects on epithelial barrier function in relation to allergic sensitisation.

We have previously published that CCL17 is produced by epithelial cells through epidermal growth factor receptordependent signalling upon downregulation of E-cadherin. ${ }^{8} \mathrm{We}$ report here that the extract that most profoundly disturbed epithelial barrier function also uniquely increased CCL17 levels in the mouse lungs. In line with our data, this specific extract 
has recently been shown to increase epidermal growth factor receptor activation in mice, contributing to $\mathrm{AHR}^{34}$ and mucus production/goblet cell hyperplasia. ${ }^{35}$

In summary, we demonstrate that allergic sensitisation to HDM does not critically involve serine/cysteine protease activity, but is related to the disruption of epithelial barrier function and pro-inflammatory epithelial responses. Our data demonstrate that HDM can induce delocalisation of E-cadherin and TJ proteins independently of serine/cysteine proteases, possibly by heat-insensitive proteases, yet the exact mechanism has to be established. It is important to further unravel these mechanisms, since the extract with the most detrimental effects on barrier function in vitro also showed increased IL-5 and CCL17 levels and induced allergic sensitisation, eosinophilic airway inflammation, AHR and goblet cell hyperplasia in vivo. Based on our data, we propose that epithelial barrier function serves as an important target for future therapeutic strategies in asthma.

Acknowledgements We thank Citeq Biologics for the kind gift of their extract and L. den Boef (Laboratory of Allergology and Pulmonary Diseases, Department of Pathology and Medical Biology, University Medical Center Groningen, Groningen, The Netherlands) for technical assistance with the Flexivent experiment. The authors also thank Professor Dr D S Postma for valuable discussions.

Funding This study was supported by a grant from the Netherlands Asthma Foundation (NAF 3.2.07.019).

\section{Competing interests None}

Contributors SP performed the cell studies, mouse experiments, analysed and interpreted the data and wrote the manuscript. MN designed and supervised the mouse experiments and edited the manuscript. TH interpreted the IHC data and contributed to the manuscript discussion. MB performed the HDM-lgE ELISA. RG assisted on the Flexivent and ear swelling experiment. AO supervised and edited the manuscript. IH coordinated and designed the studies, supervised the cell experiments and edited the manuscript.

Provenance and peer review Not commissioned; externally peer reviewed.

\section{REFERENCES}

1. Goto Y, Uchida Y, Nomura A, et al. Dislocation of E-cadherin in the airway epithelium during an antigen-induced asthmatic response. Am J Respir Cell Mol Biol 2000:23:712-18.

2. Holgate ST. The airway epithelium is central to the pathogenesis of asthma. Allergol Int 2008;57:1-10.

3. Page K, Ledford JR, Zhou P, et al. Mucosal sensitization to German cockroach involves protease-activated receptor-2. Respir Res 2010;11:62.

4. Robinson C, Kalsheker NA, Srinivasan N, et al. On the potential significance of the enzymatic activity of mite allergens to immunogenicity. Clues to structure and function revealed by molecular characterization. Clin Exp Allergy 1997;27:10-21.

5. Winter MC, Shasby SS, Ries DR, et al. PAR2 activation interrupts E-cadherin adhesion and compromises the airway epithelial barrier: protective effect of betaagonists. Am J Physiol Lung Cell Mol Physiol 2006;291:L628-35.

6. Wan H, Winton HL, Soeller C, et al. The transmembrane protein occludin of epithelial tight junctions is a functional target for serine peptidases from faecal pellets of Dermatophagoides pteronyssinus. Clin Exp Allergy 2001;31:279-94.

7. Wan H, Winton HL, Soeller C, et al. Der $\mathrm{p} 1$ facilitates transepithelial allergen delivery by disruption of tight junctions. J Clin Invest 1999;104:123-33.

8. Heijink IH, Kies PM, Kauffman HF, et al. Down-regulation of E-cadherin in human bronchial epithelial cells leads to epidermal growth factor receptor-dependent Th2 cell-promoting activity. J Immunol 2007;178:7678-85.

9. Ying S, O'Connor B, Ratoff J, et al. Thymic stromal lymphopoietin expression is increased in asthmatic airways and correlates with expression of Th2-attracting chemokines and disease severity. J Immunol 2005;174:8183-90.
10. Kauffman HF, Tamm M, Timmerman JA, et al. House dust mite major allergens De p 1 and Der $p 5$ activate human airway-derived epithelial cells by protease-dependent and protease-independent mechanisms. Clin Mol Allergy 2006;4:5.

11. Asokananthan N, Graham PT, Stewart DJ, et al. House dust mite allergens induce proinflammatory cytokines from respiratory epithelial cells: the cysteine protease allergen, Der $p 1$, activates protease-activated receptor (PAR)-2 and inactivates PAR1. J Immunol 2002;169:4572-8.

12. Ebeling C, Lam T, Gordon JR, et al. Proteinase-activated receptor-2 promotes allergic sensitization to an inhaled antigen through a TNF-mediated pathway. $J$ Immunol 2007;179:2910-17.

13. Fattouh R, Pouladi MA, Alvarez D, et al. House dust mite facilitates ovalbuminspecific allergic sensitization and airway inflammation. Am J Respir Crit Care Med 2005;172:314-21.

14. Kheradmand F, Kiss A, Xu J, et al. A protease-activated pathway underlying Th cell type 2 activation and allergic lung disease. J Immunol 2002;169:5904-11.

15. Elias JA, Homer RJ, Hamid 0, et al. Chitinases and chitinase-like proteins in $T(H) 2$ inflammation and asthma. J Allergy Clin Immunol 2005:116:497-500.

16. Hammad H, Chieppa M, Perros F, et al. House dust mite allergen induces asthma via toll-like receptor 4 triggering of airway structural cells. Nat Med 2009;15:410-16.

17. Nathan AT, Peterson EA, Chakir J, et al. Innate immune responses of airway epithelium to house dust mite are mediated through beta-glucan-dependent pathways. J Allergy Clin Immunol 2009;123:612-18.

18. Heijink IH, van Oosterhout A, Kapus A. Epidermal growth factor receptor signalling contributes to house dust mite-induced epithelial barrier dysfunction. Eur Respir $J$ 2010;36:1016-26.

19. Heijink IH, Brandenburg SM, Noordhoek JA, et al. Characterisation of cell adhesion in airway epithelial cell types using electric cell-substrate impedance sensing. Eur Respir J 2010:35:894-903.

20. Wegener J, Keese CR, Giaever I. Electric cell-substrate impedance sensing (ECIS as a noninvasive means to monitor the kinetics of cell spreading to artificial surfaces. Exp Cell Res 2000;259:158-66.

21. King C, Brennan S, Thompson PJ, et al. Dust mite proteolytic allergens induce cytokine release from cultured airway epithelium. J Immunol 1998:161:3645-51.

22. Reibman J, Hsu Y, Chen LC, et al. Airway epithelial cells release MIP-3alpha/CCL2O in response to cytokines and ambient particulate matter. Am J Respir Cell Mol Biol 2003;28:648-54.

23. Cates EC, Fattouh R, Wattie J, et al. Intranasal exposure of mice to house dust mite elicits allergic airway inflammation via a GM-CSF-mediated mechanism. J Immunol 2004:173:6384-92.

24. Gough L, Campbell E, Bayley D, et al. Proteolytic activity of the house dust mite allergen Der $\mathrm{p} 1$ enhances allergenicity in a mouse inhalation model. Clin Exp Allergy 2003;33:1159-63.

25. Nawijn MC, Hackett TL, Postma DS, et al. E-cadherin: gatekeeper of airway mucosa and allergic sensitization. Trends Immunol 2011;32:248-55.

26. De Alba J, Raemdonck K, Dekkak A, et al. House dust mite induces direct airway inflammation in vivo: implications for future disease therapy? Eur Respir $J$ 2010;35:1377-87.

27. Page K, Lierl KM, Herman N, et al. Differences in susceptibility to German cockroach frass and its associated proteases in induced allergic inflammation in mice. Respir Res 2007:8:91.

28. Finkelman FD, Hogan SP, Hershey GK, et al. Importance of cytokines in murine allergic airway disease and human asthma. J Immunol 2010;184:1663-74.

29. Xu S, Huo J, Lee KG, et al. Phospholipase Cgamma2 is critical for Dectin-1-mediated $\mathrm{Ca} 2+$ flux and cytokine production in dendritic cells. J Biol Chem 2009:284:7038-46.

30. Chun J, Prince A. TLR2-induced calpain cleavage of epithelial junctional proteins facilitates leukocyte transmigration. Cell Host Microbe 2009;5:47-58.

31. Trompette A, Divanovic S, Visintin A, et al. Allergenicity resulting from functional mimicry of a toll-like receptor complex protein. Nature 2009;457:585-8.

32. Osterlund C, Gronlund H, Polovic N, et al. The non-proteolytic house dust mite allergen Der p 2 induce NF-kappaB and MAPK dependent activation of bronchia epithelial cells. Clin Exp Allergy 2009;39:1199-208.

33. Dong L, Li H, Wang $\mathrm{S}$, et al. Different doses of lipopolysaccharides regulate the lung inflammation of asthmatic mice via TLR4 pathway in alveolar macrophages. J Asthma 2009;:46:229-33

34. Le Cras TD, Acciani TH, Mushaben EM, et al. Epithelial EGF receptor signaling mediates airway hyperreactivity and remodeling in a mouse model of chronic asthma. Am J Physiol Lung Cell Mol Physiol 2011;300:L414-21.

35. Burgel PR, Lazarus SC, Tam DC, et al. Human eosinophils induce mucin production in airway epithelial cells via epidermal growth factor receptor activation. J Immunol 2001;167:5948-54. 